\title{
A Framework of Darknet Forensics
}

Leng Tao1,2,3,4 *

1.Chinese Academy of Sciences, Institute of Information Engineering, lengtao@iie.ac.cn; 2.University of Chinese Academy of Sciences, School of Cyber Security; 3.Sichuan Police College, Department of Computer Science and Technology; 4.Sichuan Police College, Criminal Inspection Sichuan Provincial University Key Laboratory

\begin{abstract}
The dark web market is full of illegal and criminal activities such as the sale of sensitive personal information, guns, drugs, and terrorist videos. Cybercriminals use The Onion Router(TOR) browser to enter the dark web for information publishing and trading. Because the onion router browser provides privacy protection and anonymity, it is widely used. This privacy protection mode has brought great challenges to network investigators. This article aims to detect the use of the latest Tor browser, compare and analyze the evidence information contained in the registry, memory images, hard disk files, and network data packets through forensic experiments. At the same time, it compares and analyzes the different access modes of the Tor browser, and collects and uses Tor browsing. Evidence of cybercrime committed by a device is helpful to the development of electronic data forensics analysis.
\end{abstract}

\section{CCS CONCEPTS}

- Security and privacy; $\bullet$ Human and societal aspects of security and privacy;

\section{KEYWORDS}

tor browser, memory forensic, network forensic, artifacts

\section{ACM Reference Format:}

Leng Tao1,2,3,4 * and Yu Aimin1,2. 2021. A Framework of Darknet Forensics. In 2021 3rd International Conference on Advanced Information Science and System (AISS 2021) (AISS 2021), November 26-28, 2021, Sanya, China. ACM, New York, NY, USA, 6 pages. https://doi.org/10.1145/3503047.3503082

\section{INTRODUCTION}

In recent years, as the awareness of personal privacy protection has increased, network traffic is encrypted with HTTPS , and endpoint browsers provide privacy-protected modes for trace-free browsing. The dark web offers a wealth of resources that require access with a dedicated browser, such as Tor. The Tor browser is designed to achieve anonymity and protect privacy. Accessing the dark web with Tor ensures users' private information, but also increases cybercrime. The dark web market is flooded with many sales of

Permission to make digital or hard copies of all or part of this work for personal or classroom use is granted without fee provided that copies are not made or distributed for profit or commercial advantage and that copies bear this notice and the full citation on the first page. Copyrights for components of this work owned by others than ACM must be honored. Abstracting with credit is permitted. To copy otherwise, or republish, to post on servers or to redistribute to lists, requires prior specific permission and/or a fee. Request permissions from permissions@acm.org.

AISS 2021, November 26-28, 2021, Sanya, China

(C) 2021 Association for Computing Machinery.

ACM ISBN 978-1-4503-8586-2/21/11 ..\$15.00

https://doi.org/10.1145/3503047.3503082
Yu Aimin1,2

1.Chinese Academy of Sciences, Institute of Information

Engineering, yuaimin@iie.ac.cn; 2.University of Chinese Academy of Sciences, School of Cyber Security

personally identifiable information, child pornography, guns, drugs, murder buying, and terrorist activities, which poses a great challenge for cybersecurity law enforcement and requires research on forensic methods for the dark web. Previous studies have focused on Tor traffic identification and monitoring, Tor user identity studies, and some studies have analyzed Tor browser usage traces from a forensic perspective. The goal of this paper is to establish a systematic and repeatable framework for dark web forensics, the framework will involve registry forensics, hard disk file forensics, memory forensics, database forensics, network forensics, etc. The system platforms cover windows, Linux, mac os, and android. There are three challenges.1). How to form a set of frameworks for dark network forensics for multiple scenarios of cybercrime using the dark network.2) Previous research mainly focused on memory forensics, a few referred registry monitoring, Tor browser file forensics, this paper will combine darknet forensics, improve memory forensics, registry, hard disk forensics, based on the addition of network forensics. (3) Previous research verifies the privacy security of Tor browser from the perspective of experiments, and dark web forensic analysis from the perspective of forensic investigators presented as evidence that.

Our Contributions are as follows:1) Giving a complete framework for dark web forensics. 2) For the first time, we analyze Tor Browser forensics from 4 aspects (registry, memory, hard disk files, and network forensics.3) Verify the privacy-preserving features of the latest Tor browser and conduct a forensic case study from the investigator's perspective.

\section{BACKGROUND AND RELATED WORK}

Tor is a second-generation onion-routed anonymous communication system that has become the most popular and low-latency anonymous communication system. Tor provides plenty of ways to use it, making it easy to use in different scenarios.

\subsection{Tor network}

The Tor network consists of an authoritative directory server, onion routing, and onion proxy. The authoritative directory server is the core of the Tor network, responsible for maintaining node information throughout the network, such as storage and retrieval, and distributing it to Tor clients in the form of node snapshots and node descriptors. Onion routes are the foundation of the Tor network, and anonymous traffic is forwarded over anonymous communication links composed of multiple onion routes. Encrypted data is transmitted between two onion routes via a secure transport layer protocol. The onion proxy runs on the Tor client and provides a 
Table 1: Tor browser forensic

\begin{tabular}{|c|c|c|c|c|c|c|}
\hline Paper & $\begin{array}{l}\text { Registry } \\
\text { artefacts }\end{array}$ & $\begin{array}{l}\text { Memory } \\
\text { artefacts }\end{array}$ & $\begin{array}{l}\text { Hard disk } \\
\text { artefacts } \\
\text { (database) }\end{array}$ & $\begin{array}{l}\text { Network } \\
\text { Artefacts }\end{array}$ & $\begin{array}{l}\text { Windows/linux/Mac os/ } \\
\text { Android }\end{array}$ & Tor version \\
\hline Jadoon et al. 2019 [1] & $\checkmark$ & $\checkmark$ & $\checkmark$ & $x$ & Windows8.1 & Tor 7.0.2 \\
\hline W.Darcie et al. 2014 [2] & $\checkmark$ & $\checkmark$ & $x$ & $\checkmark$ & Win7 & Tor 3.6.1 \\
\hline A. Al-Khaleel et al. 2014 [3] & $\times$ & $\checkmark$ & $x$ & $x$ & Win7 & - \\
\hline A. Warren et al. 2017 [4] & $\checkmark$ & $\checkmark$ & $\checkmark$ & $x$ & Win10 & Tor5.0 \\
\hline Runa A Sandvik(2013) [5] & $\checkmark$ & $\times$ & $\checkmark$ & $x$ & $\begin{array}{l}\text { OS X } 10.8 \text {, Win } 7 \text {, } \\
\text { Debian } 6.0 \text {. }\end{array}$ & Tor2.3.25-6 \\
\hline M.-J. Chiu Huang(2018) [6] & $x$ & $\checkmark$ & $\checkmark$ & $x$ & Win10 & Tor7.5.2 \\
\hline D.Dayalamurthy (2013) [7] & $\times$ & $\checkmark$ & $x$ & $x$ & windows & Latest \\
\hline Kulm 2020 [8] & $\checkmark$ & $\checkmark$ & $\checkmark$ & $x$ & $\begin{array}{l}\text { Windows/Mac } \\
\text { Tails }\end{array}$ & New \\
\hline Vatsavayi, 2021 [9] & $x$ & $\checkmark$ & $x$ & $x$ & Win10 & - \\
\hline A. Chetry 2021 [10] & $x$ & $\checkmark$ & $x$ & $x$ & Win10 & - \\
\hline P. P. Sajan ,2021 [11] & $x$ & $\checkmark$ & $\checkmark$ & $x$ & Win10 & - \\
\hline Muir,2019 [12] & $\checkmark$ & $\checkmark$ & $\checkmark$ & $x$ & Win10 & Tor7.5.2 \\
\hline Al Barghouthy,2013 [13] & $x$ & $x$ & $\checkmark$ & $x$ & Android2.3.3 & Orweb v2.28 \\
\hline Al Barghouthy,2014 [14] & $x$ & $x$ & $\checkmark$ & $x$ & Android4.0.3 & Orwebv2.28 \\
\hline Claudia Meda ,2016 [15] & $x$ & $x$ & $\checkmark$ & $x$ & Android & - \\
\hline Ours & $\checkmark$ & $\checkmark$ & $\checkmark$ & $\checkmark$ & Win10 & Tor10.5.5 \\
\hline
\end{tabular}

Attention: $\checkmark$ delegate contain, $\times$ delegate no, - delegate no mention.

local socks proxy that establishes anonymous links and relays network traffic between the user's application and the Tor anonymous link.

\subsection{Related work}

Jadoon et al. [1] performed forensics on Tor Browser in windows 8.1, including registry, memory, hard disk footprint, etc. However, no network forensics were performed and rarely connected to dark websites. W. Darcie et al.[2] set up four virtual scenarios, registry analysis, memory forensics, and network forensics for each scenario. network forensics statistics on-network data flow and protocol layer statistics. Al-Khaleel et al.[3] considered only the Tor memory forensics scenario, which separately considered Tab page open and close state, entire Tor closed state, and close the browser for 15 minutes. Warren et al. [4] took three snapshots, Filesystem Artifacts about prefetch, hives, memory artifacts. Runa A Sandvik et al. [5] considers three object scenarios, the scope of the study is defined as a user who does not have administrative privileges or does not know how to delete Tor Browser traces, uses three virtual machines, does not use a browser to download the Tor Browser, but connects an external drive and then copies it, only the traces left behind after the Tor Browser is deleted and the system is completely shut down will be considered, and consider only the traces left by the Tor browser itself.M.-J. Chiu Huang et al. [6] start with cybercrime analysis, focusing on the different modes of different browsers. Compare Tor browser, chrome firefox, however, only memory images and cookie databases are considered. D.Dayalamurthy et al. [7] proposed a memory forensic framework for tor browser without experimental. Validation. Kulm et al.[8] considered windows and mac os operating systems, the study considered only the traces left by the Tor browser accessing the dark web in the host and designed a framework for Tor host traces study in general. Vatsavayi et al. [9] analyzed user activity using memory forensics. Chetry 2021 [10] presented the steps of an investigator's work against the dark web, the paper conforms to the general steps of an investigator, but the investigation evidence acquisition is incomplete. P. P. Sajan et al. [11] considered windows system memory, system local files. Muir,2019 et al. [12] considered both online forensic and static analysis methods. Al Barghouthy et al. [13] considered root mode and non Evidence information in both scenarios root mode and non-root mode, Only root mode can get some information. Al Barghouthy,2014 [14] compared the image extraction results of rootkit mode and Recovery mode, both results are the same. Claudia Meda et al. [15] proposed the Tor The focus of browser trace forensic analysis. Furthermore, Rathod et al. [16] discusses a framework for dark web forensics and argues that dark web forensics includes registry, network, memory, and data forensics as well as cryptocurrency wallet analysis. The related works of literature are as follows (Table 1).

\section{APPROACH}

Since many cybercriminal activities involve the dark web, such as buying and selling personal private information, purchasing drugs and guns, etc. Some criminal suspects establish dark networks and engage in illegal activities through them. From the investigator's perspective, we designed a framework for dark network forensics (Figure 1). It consists of two main components, Tor browser forensics, and dark web (web) forensics. Tor browser forensics focuses on obtaining evidence at the host side. Host forensics focuses on accessing the dark web through the Tor browser, making related 


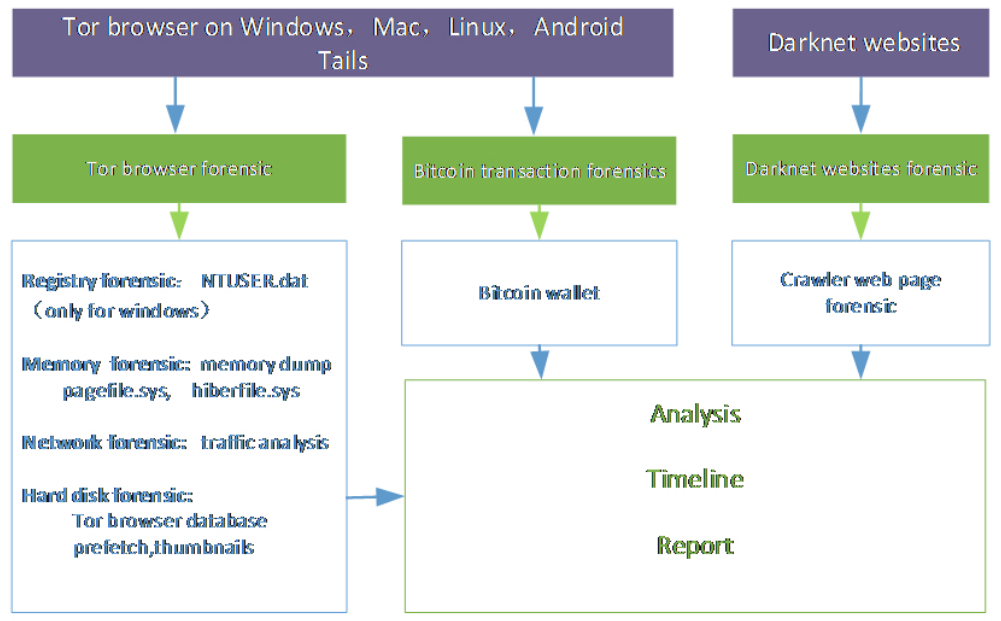

Figure 1: Darknet Forensic Framework

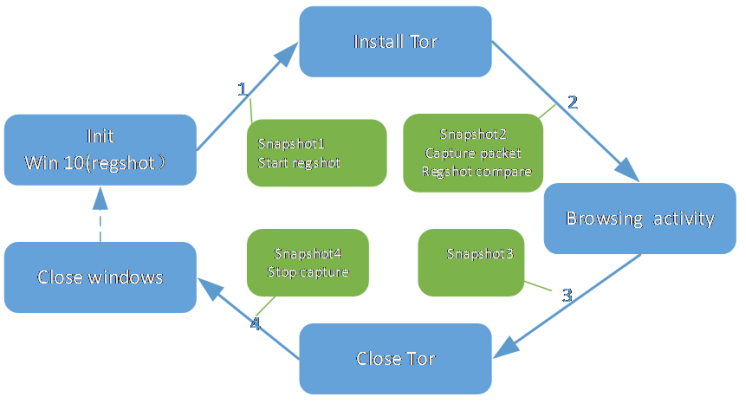

Figure 2: Pro Procedure

purchases or posting illegal information, and using bitcoin for transactions. Host forensics takes windows systems as an example, and mainly includes registry, memory, hard drive files, and network forensics. Dark network (web) forensics focuses on remote server forensics, mainly for dark web sites established by users or dark web sites that publish illegal information on the dark web, we have discussed dark web forensics in our previous article [17], so we do not discuss it in this paper. The following section focuses on the design of experiments to obtain relevant evidence at the host side.

In order to collect the complete traces of Tor browser, we designed the following experiment with a pure windows 10 virtual machine with the latest tor browser installed.

\subsection{Experiment Setup and tools}

Build a pure Win 10 virtual machine, install Tor Browser, access the explicit and dark web, close Tor Browser, and relate the windows system. Use related tools to record registry, memory, capture packets, etc. (Figure 2).The detailed process is described in section 4.2. Tools used include Vmware workstation 15 Pro, torbrowserinstall-win64-10.5.6_zh-CN, Regshot1.9.0, Volatility 2.6, Access Data FTK Imager, Bulk extractor, HxD, Rawcap, Networkminer.Registry viewer.

\subsection{Procedure}

First, prepare the win10 operating system, install the Regshot software and save the first snapshot.then, install the tor browser, use Regshot to compare the registry changes, and save the second snapshot. Third, start capture packets, use the tor browser Visit Facebook, telegram, dark web sites and save the third snapshot. Fourth, close the Tor browser and close the packet capture. The browsing modes of browsing websites include regular privacy mode and customed record browsing history mode. The browsing activity design is shown in Table 2. Memory data collection considers two modes, the browser is open, and the browser is closed.

\section{ANALYSIS AND RESULTS}

\subsection{Registry analysis}

Under win10 system, startup Regshot software, take the first snapshot, install tor browser, choose to install only and not start immediately, take the second snapshot, and compare with the first snapshot, find that the changes in the Tor browser. (Table 3)

Besides, we use AccessData Registry Viewer to analyze NTUSER.dat under the user of the win10 system. Use the Tor browser as a keyword search analysis result, find relevant information under six catalog items (Table 4). Through the registry analysis, the forensics personnel can obtain the tor browser version, installation location and installation time installed by the user.

\subsection{Memory analysis}

Memory forensics can be analyzed by volatility tools, commonly used plugins are cmdline, dlllist, dumpfiles, envars, pslist, pstree, shellbags, timeliner. Bulk extractor can find a website domain name, email, and other useful information in memory, but after Tor browser close it is less than Tor browser open (Figure 3, Figure 4), moreover, there is no user email information for login to Facebook. Therefore, at the crime scene, ensure that the suspect's computer is not closed and the tor browser is not closed for volatile data extraction. 
Table 2: Browsing activity

\begin{tabular}{|c|c|c|}
\hline Website & Activites & Accounts used on tor \\
\hline \multirow[t]{4}{*}{ facebook } & Open website & user1@gmail.com \\
\hline & Login & \\
\hline & Browsing website & \\
\hline & Download video & \\
\hline \multirow[t]{6}{*}{ telegram } & Open web app (https://web.telegram.org/)\penalty-\@M & user2 \\
\hline & Login & \\
\hline & Join group (@awlgff, @binancechinese ) & \\
\hline & Chat in the group & \\
\hline & Chat with one person & \\
\hline & Delete the message & \\
\hline \multirow[t]{3}{*}{ http://alibaba2kw6qoh6o.onion/ } & Open website & tor_user1 \\
\hline & Login & \\
\hline & Browsing website & \\
\hline
\end{tabular}

Table 3: Registry arfefacts

\begin{tabular}{|c|c|c|}
\hline S.no & Registry artefacts & Description \\
\hline 1 & $\begin{array}{l}\text { HKLM } \backslash S Y S T E M \backslash \text { ControlSet001 \Services } \backslash \\
\text { bam } \backslash \text { State } \backslash \text { UserSettings } \backslash S-1-5-21-1501606697-969712618-2041226544- \\
\text { 1002 } \backslash \text { Device } \backslash \text { HarddiskVolume3 } \backslash \text { tools } \backslash \text { torbrowser-install-win64-10.5.5_zh-CN.exe: } 6351 \text { 4C } \\
\text { 7C 5B AC D7 } 0100000000000000000000000002000000\end{array}$ & $\begin{array}{l}\text { Tor Browser configuration } \\
\text { information is included in } \\
\text { the system default } \\
\text { configuration }\end{array}$ \\
\hline 2 & $\begin{array}{l}\text { HKLM } \backslash S Y S T E M \backslash \text { CurrentControlSet } \backslash \text { Services } \backslash \text { bam } \backslash \text { State } \backslash \text { UserSettings } \backslash S-1-5-21- \\
\text { 1501606697-969712618-2041226544-1002 } \backslash \text { Device } \backslash \text { HarddiskVolume3 } \backslash \text { tools } \backslash \text { torbrowser- } \\
\text { install-win64-10.5.5_zh-CN.exe: } 6351 \text { 4C 7C 5B AC D7 } 01000000000000000000000000 \\
02000000\end{array}$ & $\begin{array}{l}\text { The current system } \\
\text { configuration contains Tor } \\
\text { Browser configuration } \\
\text { information }\end{array}$ \\
\hline 3 & 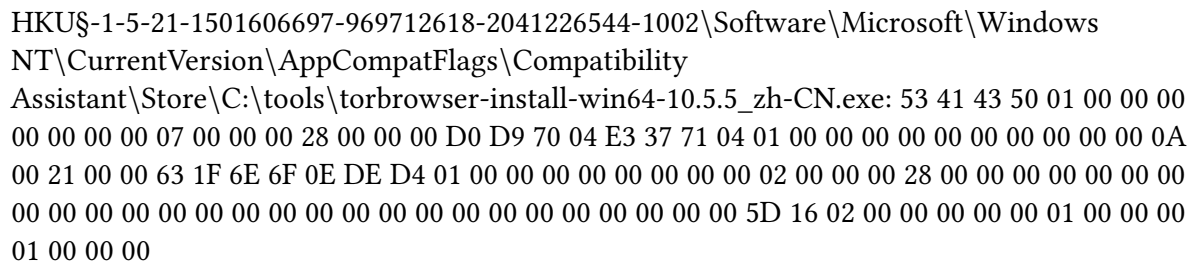 & $\begin{array}{l}\text { Indicates where the Tor } \\
\text { Browser is stored }\end{array}$ \\
\hline
\end{tabular}

Table 4: ntuser.dat artefacts

\begin{tabular}{|c|c|c|}
\hline Num & Catalog item & description \\
\hline 1 & NTUSER.DAT \Software $\backslash$ Microsoft $\backslash$ Internet & audio \\
\hline & \multicolumn{2}{|c|}{ Explorer $\backslash$ LowRegistry $\backslash$ Audio $\backslash$ PolicyConfig $\backslash$ PropertyStore $\backslash$ bc92de0a_0 } \\
\hline 2 & \multicolumn{2}{|c|}{$\begin{array}{l}\text { NTUSER.DAT } \backslash \text { Software } \backslash \text { Microsoft } \backslash \text { Windows } \backslash \text { CurrentVersion } \backslash \text { CloudStore } \backslash \text { Store } \backslash \text { Cache } \backslash \text { DefaultAccount } \backslash \text { de } \backslash 7651 \text { cac\&tore cache } \\
\text { d119-427e-b663-6b0545630146\windows.data.unifiedtile.localstartvolatiletilepropertiesmap } \backslash \text { Current }\end{array}$} \\
\hline 3 & NTUSER.DAT \Software $\backslash$ Microsoft $\backslash$ Windows $\backslash$ CurrentVersion $\backslash U F H \backslash S H C$ & $\begin{array}{l}\text { Start menu } \\
\text { lnk }\end{array}$ \\
\hline 4 & NTUSER.DAT $\backslash$ Software $\backslash$ Microsoft $\backslash$ Windows $\backslash$ Shell $\backslash$ Bags $\backslash 1 \backslash$ Desktop & Iconlayouts \\
\hline 5 & $\begin{array}{l}\text { NTUSER.DAT } \backslash \text { Software } \backslash \text { Microsoft } \backslash \text { Windows NT } \backslash \text { CurrentVersion } \backslash \text { AppCompatFlags } \backslash \text { Compatibility } \\
\text { Assistant } \backslash \text { Store }\end{array}$ & $\begin{array}{l}\text { Path for file } \\
\text { store }\end{array}$ \\
\hline 6 & NTUSER.DAT \Software \Mozilla \Firefox \Launcher & $\begin{array}{l}\text { Firefox } \\
\text { Launcher }\end{array}$ \\
\hline
\end{tabular}


Table 5: profile.default files artefacts

\begin{tabular}{llll}
\hline Analysis item & normal open & Definition open & Defintion close \\
\hline Visit history & Nothing & all websites visited & Nothing \\
Form history & Nothing & $\begin{array}{l}\text { Facebook websites form have username login } \\
\text { Tor form have no results }\end{array}$ & Nothing \\
\hline
\end{tabular}

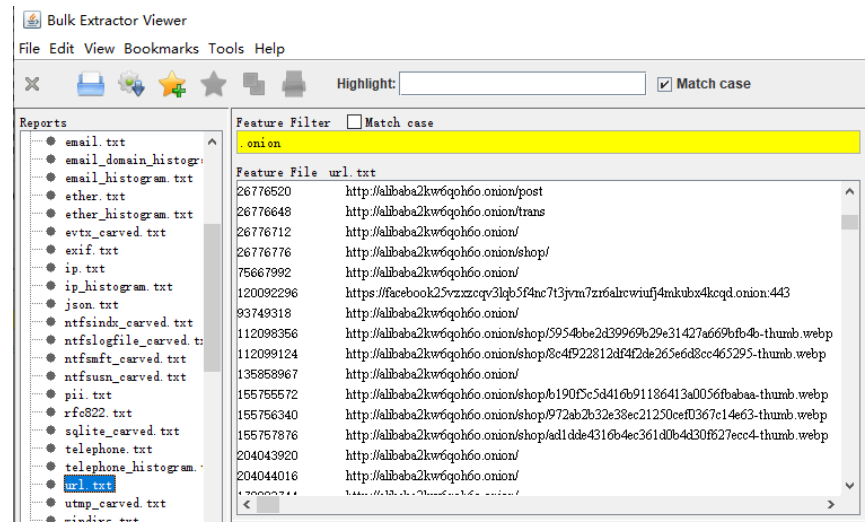

Figure 3: URL history (Tor browser opened)

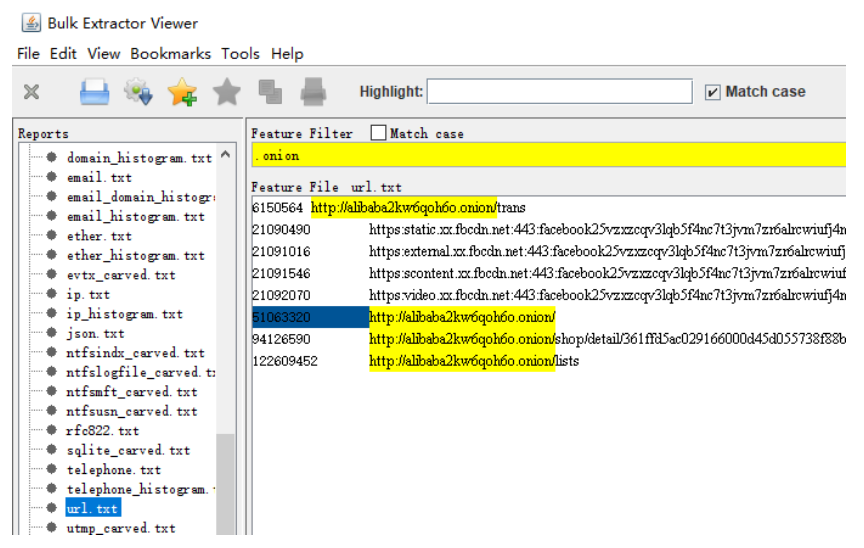

Figure 4: URL history(Tor browser closed)

\subsection{Hard disk analysis}

The image file was analyzed in [1], and information such as PUBLIC Key was found. This result was also verified in our experiment. Now we will Focus on the analysis of files such as profile.default and prefetch.

4.3.1 profile.default files. Use tor browser to visit the website, choose the default regular mode and custom two modes. In the custom mode, set to remember browsing and download history, remember to search and form history, and clear the history when you close the tor browser. Analyze the database files under the profile.default file, and the results are in Table 5. If you use the default tor standard installation mode, the tor browser installation directory database file will have no record. If you modify the installation mode and record information, you can find the browsing record in the database

4.3.2 prefetch files. Through experiments, we can find three files related to tor browser in Prefetch file, use winPrefetchView to load and view (WinPrefetchView.exe /folder F: \anwang \results $\backslash$ pf), you can get tor browser creation time, modification time, running times, and running time, As shown in Figure 5. This information can be correlated and analyzed with the case timeline.

\subsection{Network analysis}

Because Tor Browser uses a local proxy for access, it can sniff the local before it is encrypted. Use Rawcap 127.0.0.1. |torcap.pcap to capture the data packet, and then use Networkminer to analyze, the result is shown in Figure 6. If the Tor encrypted data packets are captured on the export traffic of operators, deep learning is now used to identify Tor traffic. This article will not discuss such situations.

\section{BITCOIN WALLET FORENSIC}

Bitcoin forensics mainly considers Bitcoin client file analysis, browsing Bitcoin-related websites, and relevant information in social chat messages. The Bitcoin-Qt folder mainly contains Blocks, Chanstate, Debug.log, Peers.dat, Walet.dat, etc. Here, we give a real example of a hacker using Bitcoin to conduct transactions, and after re-copying his backup wallet to the local wallet, check the transaction information. From the Figure 7 we can see the time and amount of the transaction.

\section{CONCLUSION}

In this paper, we described the composition and working principle of TOR ,Proposed a framework for dark web forensics, mainly based on the latest tor browser under the windows platform, carried out registry, memory, file, network forensics and digital wallet forensics.Tor browsers on android, mac, linux and The relationship data mining is our future work.

\section{ACKNOWLEDGMENTS}

grant sponsor by Criminal Inspection Sichuan Provincial University Key Laboratory.Grant no:2020ZD03

\section{REFERENCES}

[1] Jadoon, A. K., Iqbal, W., Amjad, M. F., Afzal, H., \& Bangash, Y. A. (2019). Forensic analysis of Tor browser: a case study for privacy and anonymity on the web. Forensic science international, 299, 59-73.

[2] Darcie, W., Boggs, R. J., Sammons, J., \& Fenger, T. (2014). Online anonymity: forensic analysis of the tor browser bundle. Technical Report, 1-34.

[3] Al-Khaleel, A., Bani-Salameh, D., \& Al-Saleh, M. I. (2014, January). On the memory artifacts of the tor browser bundle. In The International Conference on Computing 


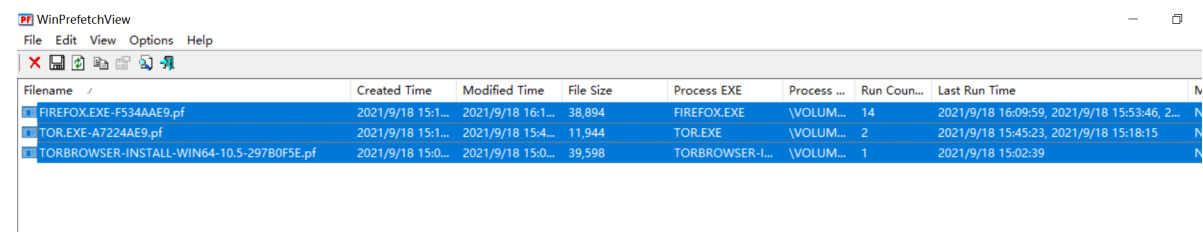

Figure 5: prefetch files artefacts

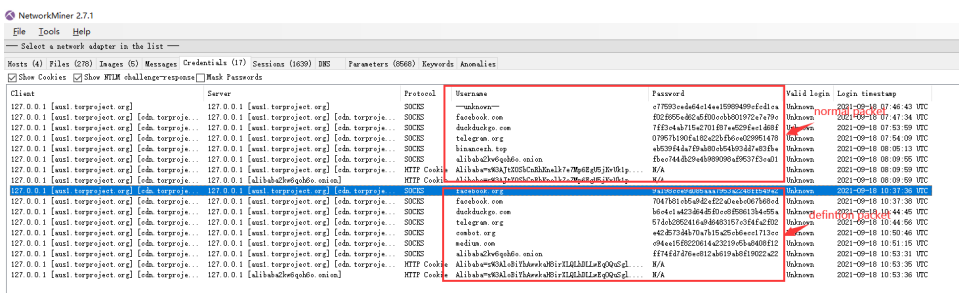

Figure 6: network artefacts

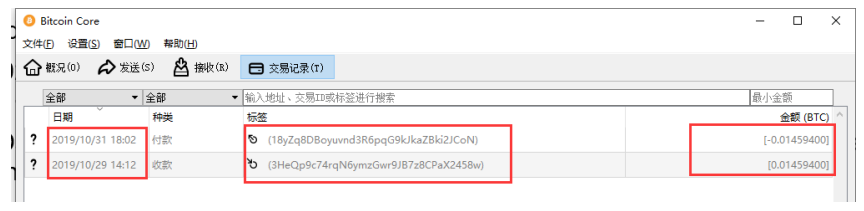

Figure 7: bitcoin wallet forensic

Technology and Information Management (ICCTIM) (p. 41). Society of Digital Information and Wireless Communication.

[4] A. Warren, Tor Browser Artifacts in Windows 10, (2017) Retrieved from SANS Institute website: https://www.sans.org/reading-room/whitepapers/foren-sics/ tor-browser-artifacts-windows-10-37642

[5] Sandvik, R. A. (2013). Forensic Analysis of the Tor Browser Bundle on OS X Linux, and Windows. Technical Report, 1-13.

[6] Huang, M. J. C., Wan, Y. L., Chiang, C. P., \& Wang, S. J. (2018, October). Tor browser forensics in exploring invisible evidence. In 2018 IEEE International Conference on Systems, Man, and Cybernetics (SMC) (pp. 3909-3914). IEEE.

[7] Dayalamurthy, D. (2013). Forensic Memory Dump Analysis And Recovery Of The Artefacts Of Using Tor Bundle Browser-The Need.

[8] Kulm, A. (2020). A Framework for Identifying Host-based Artifacts in Dark Web Investigations.
[9] Vatsavayi, V. K., \& Varma, K. S. (2021). Retrieving TOR Browser Digital Artifacts for Forensic Evidence. In Machine Intelligence and Soft Computing (pp. 265-274). Springer, Singapore.

[10] Chetry, A., \& Sharma, U. (2021). Dark web Activity on Tor-investigation challenges and retrieval of memory artifacts. In International Conference on Innovative Computing and Communications (pp. 953-964). Springer, Singapore

[11] P. P. Sajan and E. Al, "Tor Browser Forensics," Turkish Journal of Computer and Mathematics Education (TURCOMAT), vol. 12, no. 11, Art. no. 11, May 2021

[12] Al Barghouthy, N., Marrington, A., \& Baggili, I. (2013, March). The forensic investigation of android private browsing sessions using orweb. In 2013 5th International Conference on Computer Science and Information Technology (pp. 33-37). IEEE.

[13] Al Barghouthy, N., \& Marrington, A. (2014, March). A comparison of forensic acquisition techniques for android devices: a case study investigation of orweb browsing sessions. In 2014 6th International Conference on New Technologies, Mobility and Security (NTMS) (pp. 1-4). IEEE.

[14] Claudia Meda, Mattia Epifani.(2016). Study and Analysis of Orweb Anonymizer on Android Devices. DFRWS EU 2016,1-25

[15] Muir, M., Leimich, P., \& Buchanan, W. J. (2019). A forensic audit of the tor browser bundle. Digital Investigation, 29, 118-128.

[16] Rathod, D. (2017). Darknet forensics. future, 11, 12.

[17] Leng Tao, Gao Binhan, Xiong Yue, Xie Keng. Design and implementation of a monitoring platform based on dark network[J]. Network Security Technology and Applications,2021(08):26-28. 